Japan. J. Math.

Vol. 9, No. 2, 1983

\title{
Null strings and null solutions of Maxwell's equations
}

\author{
By Kiyokazu NaGatomo \\ (Received February 3, 1983)
}

\section{Introduction}

The purpose of this paper is to establish a relation between null strings and null solutions of Maxwell's equations (null electromagnetic fields). We will express Plücker's coordinates of the 2-dimensional surface swept out by a null string by means of a null electromagnetic field.

Let $(M, \eta)$ be Minkowski space with the affine coordinates $x^{\mu}, \mu=0,1,2$, 3. Throughout this paper we adopt the summation convention. Namely, we suppress the summation sign every time when the summation is to be done over an index which appears twice in the term. To raise or lower indices, we use the formulas $X_{\mu}=\eta_{\mu \nu} X^{\nu}$ and $X^{\mu}=\eta^{\mu \nu} X_{\nu}$, where $\eta_{\mu \nu}$ are the components of the metric $\eta$ and the matrix $\left(\eta^{\mu \nu}\right)$ is the inverse of the matrix $\left(\eta_{\mu \nu}\right)$.

A string is an extremal, i.e., a solution of the Euler-Lagrange equations, of the variational problem for 2 -dimensional surfaces $\Sigma: x^{\mu}\left(\tau^{1}, \tau^{2}\right)$ in $M$ :

$$
\delta\left(\int L d \tau^{1} d \tau^{2}\right)=0
$$

with Lagrangian $L=v_{\mu \nu} v^{\mu \nu}$, where we define Plücker's coordinates $v^{\mu \nu}$ of the string $\Sigma$ by $v^{\mu \nu}=\left(\partial x^{\mu} / \partial \tau^{1}\right)\left(\partial x^{\nu} / \partial \tau^{2}\right)-\left(\partial x^{\nu} / \partial \tau^{1}\right)\left(\partial x^{\mu} / \partial \tau^{2}\right)$. A string on which $L=0$ is said to be null. In Minkowski space, Maxwell's equations take the Lorentz invariant form $d F=0=d * F$ for differential 2 -forms $F$, where $*$ is the Hodge operator. If a solution $F$ of Maxwell's equations satisfies $\eta(F, F)=0$ $=\eta(F, * F)$, we call it a null electromagnetic field.

Both null strings and null electromagnetic fields are characterized by certain families of null straight lines. A string is generated by a 1-parameter family of null straight lines and a null electromagnetic field can be constructed from a 3-parameter shear-free family of null straight lines. Therefore we can apply Penrose's twistor theory to the calculation of null strings and null electromagnetic fields.

We will show that for any analytic null string there exists a null electromagnetic field $F=F_{\mu \nu} d x^{\mu} \wedge d x^{\nu}$ such that 


$$
v^{\mu \nu}\left(\tau^{1}, \tau^{2}\right)=F^{\mu \nu}\left(x\left(\tau^{1}, \tau^{2}\right)\right) .
$$

For this purpose we first prove that every 1-parameter analytic family of null straight lines can be extended to a 3-parameter shear-free family of null straight lines. In terms of twistor, this corresponds to the extension of a holomorphic curve in the projective 3 -space $\boldsymbol{P}^{3}(C)$ to a 2 -dimensional holomorphic surface in $\boldsymbol{P}^{3}(C)$. Therefore this shear-free family is not uniquely determined. Next we construct a null electromagnetic field from this extended 3-parameter shear-free family of null straight lines. After some modification, we can find a null electromagnetic field which satisfies (1). Thus we can construct a null electromagnetic field subject to (1) from any 3-parameter shear-free family of null straight lines which is constructed from the null string.

Our main theorem is as follows.

TheOREM A. Let a 2-dimensional surface $\Sigma: x^{\mu}\left(\tau^{1}, \tau^{2}\right)$ in Minkowski space be an analytic null string and let $x_{(0)}$ be a point on $\Sigma$. Then on some neighbourhood $U_{0}$ of $x_{(0)}$, a null electromagnetic field $F=F_{\mu \nu} d x^{\mu} \wedge d x^{\nu}$ such that

$$
v^{\mu \nu}\left(\tau^{1}, \tau^{2}\right)=F^{\mu \nu}\left(x\left(\tau^{1}, \tau^{2}\right)\right), \quad x\left(\tau^{1}, \tau^{2}\right)=\left(x^{\mu}\left(\tau^{1}, \tau^{2}\right)\right) \in U_{0}
$$

exists.

Kastrup [7], [8], Rinke [11] and Kastrup-Rinke [9] studied strings $x^{\prime \prime}\left(\tau^{1}, \tau^{2}\right)$ on which $L=-1$ (spacelike strings). Rinke [11] proved the existence of a closed 2-form $F=F_{\mu \nu} d x^{\mu} \wedge d x^{\nu}$ which satisfies $\eta(F, F)=-1, \eta(F, * F)=0$ and (1). His proof is based on the theory of Carathéodory, which is a multidimensional analogue of the Hamilton-Jacobi theory, especially on embedding theorem of extremals into geodesic fields (see, for example, [1], [13], [16]). In the case of null strings Lagrangian $L$ is degenerate and therefore embedding theorem can not be applied.

By a direct calculation we have:

THeOREM B. Let $F=F_{\mu \nu} d x^{\mu} \wedge d x^{\nu}$ be a null electromagnetic field. If a 2dimensional surface $\Sigma: x^{\mu}\left(\tau^{1}, \tau^{2}\right)$ in Minkowski space satisfies (1), then $\Sigma$ is a null string.

We have shown in [5] that null electromagnetic fields can be constructed from holomorphic functions on an open set in $\boldsymbol{P}^{3}(C)$. Let $F$ be a null electromagnetic field. Since $* F$ is closed and rank 2, there exist functions $T_{1}(x)$, $T_{2}(x)$ such that $* F=d T_{1} \wedge d T_{2}$. Let $\Sigma$ be a surface defined by $T_{i}=c_{i}(i=1,2)$, $c_{i}$ being constants. Then we can take coordinates $\tau^{1}, \tau^{2}$ of $\Sigma$ such that $\Sigma: x^{\mu}\left(\tau^{1}, \tau^{2}\right)$ satisfies (1). By theorem $B, \Sigma: x^{\mu}\left(\tau^{1}, \tau^{2}\right)$ is a null string.

In our proof of theorem $A$, the analycity of $\Sigma$ is essential. However, in 
theorem $B$ null string $\Sigma$ is not necessarily analytic and therefore the analycity of $\Sigma$ in theorem $A$ may be omitted.

In section 1 we give geometrical properties of null strings. In section 2 we introduce a quasi-orthonormal basis and give Robinson's method of constructing null electromagnetic fields. In section 3 we prove that every 1parameter family of null straight lines can be extended to a 3-parameter shear-free family of null straight lines. The main theorem will be proved in section 4. The auther would like to express his hearty thanks to Professor S. Tanaka and Professor T. Tsujishita for their useful comments on this work. Finally we should mention that the summary of the contents of this paper is found in Proc. Japan Acad. 59, Ser. A (1983), 136-138.

\section{$\S 1$. Null strings}

We study geometric properties of manifolds swept out by null strings.

Let $(M, \eta)$ be the Minkowski space, namely, $M$ is the affine 4-space $\boldsymbol{R}^{4}$ with Cartesian coordinates $x^{0}, x^{1}, x^{2}, x^{3}$ and $\eta$ is the metric form on $M$ defined by $\eta=\left(d x^{0}\right)^{2}-\left(d x^{1}\right)^{2}-\left(d x^{2}\right)^{2}-\left(d x^{3}\right)^{2}$. Let $x^{\mu}=x^{\mu}\left(\tau^{1}, \tau^{2}\right), \mu=0,1,2,3$ be the 2 dimentional surface $\Sigma$ in the Minkowski space swept out by a string. The action $A$ for the string motion in Schild form [15] can be written as

$$
A=\int L d \tau^{1} d \tau^{2}
$$

with Lagrangian $L=v_{\mu \nu} \nu^{\mu \nu}$ (summation convention), where Plücker's coordinates $v^{\mu \nu}$ of $\Sigma$ are defined by

$$
v^{\mu \nu}=\frac{\partial x^{\mu}}{\partial \tau^{1}} \frac{\partial x^{\nu}}{\partial \tau^{2}}-\frac{\partial x^{\nu}}{\partial \tau^{1}} \frac{\partial x^{\mu}}{\partial \tau^{2}}
$$

The Euler-Lagrange equations for (1.1) are

$$
\frac{\partial}{\partial \tau^{1}}\left(v_{\mu \nu} \frac{\partial x^{\nu}}{\partial \tau^{2}}\right)-\frac{\partial}{\partial \tau^{2}}\left(v_{\mu \nu} \frac{\partial x^{\nu}}{\partial \tau^{1}}\right)=0, \mu=0,1,2,3 .
$$

The solutions of equations (1.2) are called strings. We can verify that (1.2) implies $v_{\mu_{\nu}} \nu^{\mu \nu}=$ constant. Therefore we have three classes of strings.

Definition. A string $\Sigma: x^{\mu}\left(\tau^{1}, \tau^{2}\right)$ is said to be timelike, null, or spacelike depending on whether $v_{\mu \nu} v^{\mu \nu}$ is positive, zero, or negative, respectively.

Rinke [11] discussed timelike and spacelike strings. Here we consider null strings.

A 2-dimensional surface with Plücker's coordinates $v^{\mu \nu}$, such that the relation $v_{\mu \nu} v^{\mu \nu}=0$ is valid, is said to be null. Let $\Sigma: x^{\mu}\left(\tau^{1}, \tau^{2}\right)$ be a null 2 - 
dimensional surface in $M$. We denote the embedding of $\Sigma$ into $M$ by $\iota$. Then, $g=\iota^{*} \eta$ (the pull back of $\eta$ ), is degenerate. Therefore there exists a vector field $X=X^{i}\left(\partial / \partial \tau^{i}\right)$, which is unique up to multiple, such that $g_{i j} X^{i}=0$. Let $\sigma=\sigma^{\mu}\left(\partial / \partial x^{\mu}\right)$ be the vector tangent to $\Sigma$ defined by $\sigma=\iota_{*} X$, then

$$
\begin{aligned}
v_{\mu \nu} \sigma^{\nu} & =v_{\mu \nu}\left(X^{i} \frac{\partial x^{\nu}}{\partial \tau^{i}}\right)=\left(\eta_{\mu \rho} \eta_{\nu \varepsilon} \varepsilon^{k j} \frac{\partial x^{\rho}}{\partial \tau^{k}} \frac{\partial x^{\varepsilon}}{\partial \tau^{j}} \frac{\partial x^{\nu}}{\partial \tau^{i}}\right) X^{i} \\
& =\left(g_{i j} X^{i}\right)\left(\eta_{\mu \rho} \varepsilon^{k j} \frac{\partial x^{\rho}}{\partial \tau^{k}}\right)=0,
\end{aligned}
$$

where $\varepsilon^{k j}$ is the alternating symbol: $\varepsilon^{11}=\varepsilon^{22}=0, \varepsilon^{12}=-\varepsilon^{21}=1$. Conversely, if $\sigma=\iota_{*} X$ satisfies $v_{\mu \nu} \sigma^{\nu}=0$, then we have $g_{i j} X^{i}=0$. A vector $\sigma=\sigma^{\mu}\left(\partial / \partial x^{\mu}\right)$ is tangent to $\Sigma$ if and only if $* v_{\mu \nu} \sigma^{\nu}=0$ holds, where $*$ is the Hodge operator. Thus we have:

Proposition 1.1. Let $\Sigma$ be a null 2-dimensional surface in $M$. If a vector field $X$ on $\Sigma$ satisfies $g_{i j} X^{i}=0$, then for $\sigma=\iota_{*} X$

$$
\begin{aligned}
& v_{\mu \nu} \sigma^{\nu}=0, \\
& * v_{\mu \nu} \sigma^{\nu}=0
\end{aligned}
$$

hold. Conversely, any vector $\sigma$ subject to (1.3) and (1.4) has the form $\sigma=\iota_{*} X$, where $X$ is a vector field on $\Sigma$ such that $g_{i j} X^{i}=0$.

REMARK. The conditions (1.3) and (1.4) determine $\sigma$ uniquely up to multiple.

We can easily verify:

Corollary 1.2. If a vector $\sigma$ satisfies (1.3) and (1.4), then $\sigma$ is null, i.e., $\sigma_{\mu} \sigma^{\mu}=0$.

Let $\Sigma: x^{\prime \prime}\left(\tau^{1}, \tau^{2}\right)$ be a null string. Since $\Sigma$ is the null 2-dimensional surface, there exists a vector field $X$ on $\Sigma$ such that $\sigma=\iota_{*} X$ satisfies $v_{\mu \nu} \sigma^{\nu}=0$, $* v_{\mu \nu} \sigma^{\nu}=0$. The next theorem gives a geometrical characterization of null strings.

THEOREM 1.3. (Schild [15]). Let $\Sigma: x^{\mu}\left(\tau^{1}, \tau^{2}\right)$ be a null 2-dimensional surface in $M$ and $\sigma$ is a vector field on $\Sigma$ satisfying (1.3) and (1.4). Then $\Sigma$ is a null string if and only if all the integral curves of $\sigma$ are null straight lines.

\section{$\S 2$. Null electromagnetic fields}

Here we show that geodesic shear-free null vector fields determine null electromagnetic fields. 
A differential 2-form $F=F_{\mu \nu} d x^{\mu} \wedge d x^{\nu}$ on an open set in $M$ is said to be null if $F_{\mu \nu} F^{\mu \nu}=0$ and $F_{\mu \nu} * F^{\mu \nu}=0$. Similarly as in the case of null strings, for any real null 2 -form $F$, equations

$$
\begin{aligned}
F_{\mu \nu} n^{\nu} & =0, \\
* F_{\mu \nu} n^{\nu} & =0
\end{aligned}
$$

define a real null vector field $n=n^{\mu}\left(\partial / \partial x^{\mu}\right)$ up to multiple. Conversely, for any null vector field $n=n^{\mu}\left(\partial / \partial x^{\mu}\right)$, there exists a real null 2 -form $F=F_{\mu \nu} d x^{\mu} \wedge$ $d x^{\nu}$ satisfying (2.1) and (2.2). Let $F=F_{\mu \nu} d x^{\mu} \wedge d x^{\nu}$ be a real null 2-form subject to (2.1) and (2.2). Then any 2 -form $\hat{F}=\hat{F}_{\mu \nu} d x^{\mu} \wedge d x^{\nu}$ defined by (2.1) and (2.2) has the form

$$
\hat{F}_{\mu \nu}-i * \hat{F}_{\mu \nu}=e^{w}\left(F_{\mu \nu}-i * F_{\mu \nu}\right),
$$

where $w$ is an appropriate complex scalar. To see this, we introduce a quasi-orthonormal basis of tangent space (Newman and Penrose [6]). This plays an important role in section 4 .

A complex quasi-orthonormal basis $\{n, l, m, \bar{m}\}$ is defined by the equations

$$
\eta^{\mu \nu}=n^{\mu} l^{\nu}+n^{\nu} l^{\mu}-\left(\bar{m}^{\mu} m^{\nu}+\bar{m}^{\nu} m^{\mu}\right),
$$

here $n=n^{\mu}\left(\partial / \partial x^{\mu}\right)$ and $l=l^{\mu}\left(\partial / \partial x^{\mu}\right)$ are real vector fields, while $m=m^{\mu}\left(\partial / \partial x^{\mu}\right)$ is complex and $\bar{m}=\bar{m}^{\mu}\left(\partial / \partial x^{\mu}\right)$ is the complex conjugate of $m$. The relation (2.4) holds if and only if the quasi-orthonormality relations

$$
n^{\mu} l_{\mu}=-\bar{m}^{\mu} m_{\mu}=1, \quad n^{\mu} n_{\mu}=l^{\mu} l_{\mu}=m^{\mu} m_{\mu}=n^{\mu} m_{\mu}=l^{\mu} m_{\mu}=0
$$

hold.

We will adopt a certain quasi-orthonormal basis which is useful for our calculation.

Proposition 2.1 (Cox and Flaherty [2]). Let $n=n^{\mu}\left(\partial / \partial x^{\mu}\right)$ be a real nonzero null vector field. Then there exist vector fields $l=l^{\mu}\left(\partial / \partial x^{\mu}\right)$ and $m=$ $m^{\mu}\left(\partial / \partial x^{\mu}\right)$ such that $\{n, l, m, \bar{m}\}$ is a quasi-orthonormal basis.

For a proof see [2] and [4].

If we exchange $m=m^{\mu}\left(\partial / \partial x^{\mu}\right)$ for $\bar{m}=\bar{m}^{\mu}\left(\partial / \partial x^{\mu}\right)$, we have another quasiorthonormal basis. We choose one of these as follows.

LEMMA 2.2. Let $\omega=\omega_{\mu \nu} d x^{\mu} \wedge d x^{\nu}$ be a complex 2-form defined by $\omega_{\mu \nu}=n_{\mu} m_{\nu}$ $-n_{\nu} m_{\mu}$. Then $* \omega=i \omega$ or $* \omega=-i \omega$.

Proof. By the definition of the Hodge operator, $* \omega$ has the form

$$
* \omega_{\mu \nu}=\varepsilon_{\mu \nu \rho \varepsilon} n^{\rho} m^{\varepsilon},
$$


where $\varepsilon_{\mu \nu \rho \varepsilon}$ is the alternating symbol. Since $* \omega_{\mu \nu} n^{\nu}=0$ and $* \omega_{\mu \nu} m^{\nu}=0$, we have

$$
* \omega_{\mu \nu}=h\left(n_{\mu} m_{\nu}-n_{\nu} m_{\mu}\right)
$$

with some function $h$. The quasi-orthonormality relations (2.5) imply $h=$ $-* \omega_{\mu \nu} l^{\mu} \bar{m}^{\nu}$.

Therefore

$$
h=-\varepsilon_{\mu \nu \rho \varepsilon} l^{\mu} \bar{m}^{\nu} n^{\rho} m^{\varepsilon}=\varepsilon_{\mu \nu \rho \varepsilon} l^{\mu} \bar{m}^{\nu} m^{\rho} n^{\varepsilon} .
$$

Define a matrix $H$ by

$$
H={ }^{t}\left[l^{\mu} \bar{m}^{\mu} m^{\mu} n^{\mu}\right]
$$

Then $h$ has the expression

$$
h=\operatorname{det}(H) .
$$

The quasi-orthonormality relations (2.5) are equivalent to

$$
H \eta^{t} H=\left(\begin{array}{rrrr}
0 & 0 & 0 & 1 \\
0 & 0 & -1 & 0 \\
0 & -1 & 0 & 0 \\
1 & 0 & 0 & 0
\end{array}\right),
$$

where $\eta=\left(\eta_{\mu \nu}\right)$. Thus we have $(\operatorname{det} H)^{2}=-1$ and $h=i$ or $-i$.

Q.E.D.

Replacing $m$ by $\bar{m}$ if necessary, we can assume that $\omega$ is self dual, namely, $* \omega=i \omega$. From now on we use this quasi-orthonormal basis.

A real differential 2-form $F=F_{\mu \nu} d x^{\mu} \wedge d x^{\nu}$ subject to (2.1) takes the form

$$
F_{\mu \nu}=a\left(n_{\mu} m_{\nu}-n_{\nu} m_{\mu}\right)+b\left(n_{\mu} \bar{m}_{\nu}-n_{\nu} \bar{m}_{\mu}\right)+c\left(m_{\mu} \bar{m}_{\nu}-m_{\nu} \bar{m}_{\mu}\right)
$$

with certain functions $a, b, c$. Since $F$ is a real form, we have $b=\bar{a}$ and $\bar{c}=$ -c. By Lemma 2.2,

$$
* F_{\mu \nu}=a i\left(n_{\mu} m_{\nu}-n_{\nu} m_{\mu}\right)-b i\left(n_{u} \bar{m}_{\nu}-n_{\nu} \bar{m}_{\mu}\right)+c \varepsilon_{\mu \nu \varepsilon \rho} m^{\varepsilon} \bar{m}^{\rho}
$$

and we have

$$
\left(* F_{\mu \nu} n^{\nu}\right) l^{\mu}=c \varepsilon_{\mu \nu \varepsilon \rho} l^{\mu} n^{\nu} m^{\varepsilon} \bar{m}^{\rho}=i c .
$$

Equation (2.2) implies $c=0$. Thus a real differential 2-form $F$ satisfies equations (2.1) and (2.2) if and only if

$$
F_{\mu \nu}=a\left(n_{\mu} m_{\nu}-n_{\nu} m_{\mu}\right)+\bar{a}\left(n_{\mu} \bar{m}_{\nu}-n_{\nu} \bar{m}_{\mu}\right) .
$$

More simply we can write this as 


$$
F_{\mu \nu}-i * F_{\mu \nu}=2 a\left(n_{\mu} m_{\nu}-n_{\nu} m_{\mu}\right) .
$$

Consequently equations (2.1) and (2.2) determine a real nonzero null 2-form, which is unique up to transformations (2.3).

A real null differential 2-form $F=F_{\mu \nu} d x^{\mu} \wedge d x^{\nu}$ is said to be a null electromagnetic field if $d F=0$ and $d * F=0$. Let $F$ be a null electromagnetic field and $n=n^{\mu}\left(\partial / \partial x^{\mu}\right)$ be a null vector field which satisfies (2.1) and (2.2). Then $n=n^{\mu}\left(\partial / \partial x^{\mu}\right)$ is geodesic, i.e.,

$$
\left\{n_{[\mu ; \nu]}-i *\left(n_{[\mu ; \nu]}\right]\right\} n^{\nu}=\left\{(1 / 2) n_{; \nu}^{\nu}+\zeta\right\} n_{\mu}
$$

with a complex function $\zeta$, and shear-free, i.e.,

$$
2 n^{\mu}{ }^{\nu} n_{(\mu ; \nu)}=\left(n_{; \nu}^{\nu}+\zeta+\bar{\zeta}\right)^{2}+(\zeta+\bar{\zeta})^{2}
$$

where square and round brackets denote skew symmetrization and symmetrization, respectively (Robinson [12]). Here we write the components of the covariant derivative of $n^{\mu}$ as $n_{; \nu}^{\mu}$.

Conversely, if a vector field $n=n^{\mu}\left(\partial / \partial x^{\mu}\right)$ is null, geodesic and shear-free, we can construct null electromagnetic field which satisfies (2.1) and (2.2) by a nice choice of the function $a$ in (2.6). The process of this construction is as follows (see also Robinson [12]).

Let $n=n^{\mu}\left(\partial / \partial x^{\mu}\right)$ be a nonzero null, geodesic and shear-free vector field and let $F=F_{\mu \nu} d x^{\mu} \wedge d x^{\nu}$ be a real 2-form such that $F_{\mu \nu} n^{\nu}=0$ and $* F_{\mu \nu} n^{\nu}=0$. Then (2.1) and (2.2) are also valid for $F_{\mu \nu ; \rho} n^{\rho}$ and there exists a complex function $z$ such that

$$
\left(F_{\mu \nu}-i * F_{\mu \nu}\right)_{;} n^{\rho}=z\left(F_{\mu \nu}-i * F_{\mu \nu}\right) .
$$

The mapping (2.3) transforms $z$ to $z+w,{ }_{, \mu} n^{\mu}$. Therefore we can normalize $F$ by demanding that

$$
\left(F_{\mu \nu}-i * F_{\mu \nu}\right)_{; \rho} n^{\rho}=\zeta\left(F_{\mu \nu}-i * F_{\mu \nu}\right) .
$$

We call this 2-form $F$ a normally propagated field. A normally propagated field is determined up to the transformation (2.3) defined by $w$ such that $w_{, \mu} n^{\mu}=0$. In view of (2.7) and (2.8), normally propagated fields satisfy $J_{[\mu} n_{\nu]}$ $=0=* J_{[\mu} n_{\nu]}$, which is a necessary and sufficient condition for the existence of a scalar $b$ such that

$$
J_{\mu}-i * J_{\mu}=b n_{\mu},
$$

where $J^{\mu}=F^{\mu \nu} ;$ and $* J^{\mu}=* F_{; \nu}^{\mu \nu}$. Furthermore, (2.7), (2.10) and the identities $J^{\mu}{ }_{; \mu}=0=* J^{\mu}{ }_{; \mu}$ imply that

$$
J_{\mu ; \nu} n^{\nu}=(\zeta+\bar{\zeta}) J_{\mu}, \quad * J_{\mu ; \nu} n^{\nu}=(\zeta+\bar{\zeta}) * J_{\mu} .
$$


Under the transformation (2.3) defined by $w$ such that

$$
\begin{gathered}
w,_{\pi} n^{\mu}=0, \\
w,_{\mu} m^{\mu}+(b / 2 a)=0,
\end{gathered}
$$

the transformed 2-form satisfies $J^{\mu}=0=*^{\mu}$, which are equivalent to $d F=0$ $=d * F$.

Equation (2.11) is the integrability condition for the system of equations (2.12) and (2.13) in the sense mentioned below. By taking coordinates $\left(x^{\lambda}, x^{3}\right)$, $\lambda=0,1,2$ such that $n^{\mu}=\delta_{4}^{\mu}$, we see from (2.11) that if $J^{\mu}=* J^{\mu}=0$ is valid for one value of $x^{3}$, then they are satisfied for all $x^{3}$ in some open set in $M$. Equation (2.12) means that $w$ is independent of $x^{3}$. After the transformation (2.3) defined by $w$ which is independent of $x^{3}$, equation (2.11) also holds. If $w$ is a solution of equation (2.13) on $\left(x^{\lambda}\right)$ space of constant $x^{3}$, then the transformed 2-form is a null electromagnetic field. Equation (2.13) can be solved on $\left(x^{\lambda}\right)$ space of constant $x^{3}$. Finally we remark that if $F=F_{\mu \nu} d x^{\mu} \wedge d x^{\nu}$ is a null electromagnetic field, then equation (2.13) takes the form

$$
w,{ }_{\mu} m^{\mu}=0 \text {. }
$$

\section{$\S 3$. Geodesic shear-free null congruences}

We know already that for any null string $\Sigma$ there exists null vector field $\sigma$ on $\Sigma$. In this section we prove that we can extend $\sigma$ to the vector field on an open set in $M$ which is null, geodesic and shear-free.

Consider a null satraight line $L$ in Minkowski space. Let $l^{\mu}$ be the position vector of a point $P$ on $L$ and let $n^{\mu}$ be a future-pointing tangent vector to $L$. Define $m^{\mu \nu}$ by

$$
m^{\mu \nu}=l^{\mu} n^{\nu}-n^{\mu} l^{\nu}
$$

Since $L$ is null,

$$
n^{\mu} n_{\mu}=0
$$

and there are consistency relations for (3.1)

$$
\varepsilon_{i j k l} m^{i j} n^{k}=0 .
$$

The ratios of the ten quantities $\left(n^{\mu}, m^{\mu \nu}\right)$ subject to (3.2) and (3.3) uniquely determine a null straight line $L$. Using spinor we can simplify the description of the null line $L$. The translation from tensor to spinor is achieved by using a quantity $\sigma_{\mu}^{M M^{\prime}}$ [a Hermitian $(2 \times 2)$ matrix for each $\mu$ ] and its inverse, $\sigma_{M M}^{\mu}$, subject to

$$
\sigma_{\mu}^{M M^{\prime}} \sigma_{\nu}^{N N^{\prime}} \varepsilon_{M N} \varepsilon_{M M^{\prime} N^{\prime}}=g_{\mu \nu}, \quad \sigma_{\mu}^{M M^{\prime}} \sigma_{M M}^{\nu} M^{\prime}=\delta^{\nu}{ }_{\mu} .
$$


The $\varepsilon_{M N}, \varepsilon_{H^{\prime} N}$, are the alternating symbols and are used for raising and lowering spinor indices:

$$
\xi^{A} \varepsilon_{A B}=\xi_{B}, \quad \varepsilon^{A B} \xi_{B}=\xi^{A},
$$

i.e.,

$$
\xi^{0}=\xi_{1}, \quad \xi^{1}=-\xi_{0}
$$

and similarly for primed indices. Any tensor (e.g., $X^{\mu \nu}$ ) is transformed to a spinor, which is written by using the same base symbol, but with each tensor index being replaced by the corresponding pair of spinor indices, e.g.,

$$
X^{\mu \nu} \longrightarrow X^{M M^{\prime} N N^{\prime}}=X^{\mu \nu} \sigma_{\mu}^{M M^{\prime}} \sigma_{\nu}^{N_{\nu}^{+} N^{\prime}} .
$$

Under complex conjugation, the roles of primed and umprimed indices are interchanged, so that the reality of tensor is expressed as the Hermiticity of spinor. In Minkowski space we can choose $\sigma_{\mu \mu}^{M M^{\prime}}$ as follows:

$$
\begin{aligned}
\left(\sigma_{0}^{M M^{\prime}}\right)=\left[\begin{array}{ll}
1 & 0 \\
0 & 1
\end{array}\right], & \left(\sigma_{2}^{M M^{\prime}}\right)=\left[\begin{array}{ll}
0 & 1 \\
1 & 0
\end{array}\right] \\
\left(\sigma_{1}^{M M^{\prime}}\right)=\left[\begin{array}{rr}
1 & 0 \\
0 & -1
\end{array}\right], & \left(\sigma_{3}^{M M^{\prime}}\right)=\left[\begin{array}{rr}
0 & i \\
-i & 0
\end{array}\right] .
\end{aligned}
$$

The spinor translation of a complex null vector $a^{\mu}$ (i.e., $a_{\mu} a^{\mu}=0$ ) has the form

$$
a^{\prime \prime} \longrightarrow a^{M M^{\prime}}=b^{M} c^{M^{\prime}} .
$$

If $a^{\mu}$ is real and future pointing, then we can take $c^{M M^{\prime}}$ to be the complex conjugate of $b^{M^{\prime}}$, i.e.,

$$
a^{\mu} \longrightarrow a^{M M^{\prime}}=b^{M} \bar{b}^{M^{\prime}} .
$$

Let us now represent, in spinor terms, the quantities $n^{n}, m^{\mu \nu}$, which define the null line $L$. We have

$$
n^{\mu} \longrightarrow n^{M M^{\prime}}=\xi^{M \bar{\xi}^{M \prime}}
$$

and, from (3.1)

$$
m^{\mu \nu} \longrightarrow l^{M M^{\prime}} \xi^{N} \bar{\xi}^{N^{\prime}}-\xi^{M \bar{\xi}^{M^{\prime}} l^{N N^{\prime}}}=i \varepsilon^{M N} \eta^{\left(M^{\prime}\right.} \xi^{\left.N^{\prime \prime}\right)}-i \eta^{(M H} \xi^{M)} \varepsilon^{M^{\prime} N^{\prime}},
$$

where

$$
\eta_{A^{\prime}}=-i \xi^{A} l_{A A^{\prime}}
$$

(Round brackets denote symmetrization.) 
The null line $L$ corresponds to the three ratios of the four complex quantities

$$
L^{0}=\xi^{0} . \quad L^{1}=\xi^{1}, \quad L^{2}=\eta_{0^{\prime}}, \quad L^{3}=\eta_{1^{\prime}},
$$

which we write

$$
\left(L^{\alpha}\right)=\left(\xi^{A}, \eta_{A^{\prime}}\right)
$$

Since the reality of the vector $l^{\mu}$ implies $l_{A A^{\prime}}$ is hermitian,

$$
\operatorname{Re}\left(\xi^{A} \bar{\eta}_{A}\right)=0
$$

Provided that $\xi^{A} \neq 0$, condition (3.7) is also sufficient to ensure the existence of a null line $L$ corresponding to $\xi^{A}$ and $\eta_{A^{\prime}}$ (Penrose [10]).

Let $C^{4}$ be the complex 4 -space with coordinates $z^{\alpha}, \alpha=1,2,3,4$ and let $\Phi$ be the hermitian form on $C^{4}$ defined by

$$
\Phi=\left(\begin{array}{llll}
0 & 0 & 1 & 0 \\
0 & 0 & 0 & 1 \\
1 & 0 & 0 & 0 \\
0 & 1 & 0 & 0
\end{array}\right) .
$$

We denote the pair $\left(C^{4}, \Phi\right)$ by $T$, the space of twistors. Let

$$
\begin{gathered}
T^{+}=\left\{z \in C^{4}: \Phi(z, z)>0\right\}, \\
T^{0}=\left\{z \in C^{4}: \Phi(z, z)=0\right\}, \\
T^{-}=\left\{z \in C^{4}: \Phi(z, z)<0\right\} .
\end{gathered}
$$

We call these positive, null, and negative twistors, respectively. It follows that $T=T^{+} \cup T^{0} \cup T^{-}$and that $T^{0}$ is a real 7-dimensional cone in $C^{4}$. Equation (3.7) implies that the spinor $\left(L^{\alpha}\right)=\left(\xi^{A}, \eta_{A^{\prime}}\right)$ corresponding to a null line $L$ is a null twistor.

Since a null line $L$ is determined by the three complex ratios $L^{0}: L^{1}: L^{2}$ : $L^{3}$, where $\left(L^{\alpha}\right)$ is a null twistor, every null line is identified with an element of projective 3 -space $P^{3}(C)$. The subspace in $P^{3}(C)$ corresponding to null lines is

$$
N=\left\{[z] \in \boldsymbol{P}^{3}(C): \Phi(z, z)=0, z \in C^{4}\right\}
$$

where $[z]$ denotes the equivalent class of $z \neq 0 \in C^{4}$.

If a vector field $n=n^{\mu}\left(\partial / \partial x^{\mu}\right)$ is null, geodesic and shear-free, the set of all integral curves of $n$ is a 3-parameter family of null straight lines. We call this a shear-free null congruence. In terms of twistors, the null twistors corresponding to the vector field $n$, form a real 3 -dimensional submanifold 
in $\boldsymbol{P}^{3}(C)$, which we also call a shear-free null congruence. We can characterize shear-free null congruences using Kerr's theorem whose proof is found in Penrose [10].

TheOREm 3.1 (Kerr). If a congruence of null lines in $M$ is representable in $\boldsymbol{P}^{3}(C)$ as the intersection of $N$ with a holomorphic 2-dimensional submanifold in $\boldsymbol{P}^{3}(C)$, then it is shear-free.

Using Kerr's theorem we have:

THEOREM 3.2. Let $\Sigma$ be a null string and let $\sigma$ be an associated null vector field on $\Sigma$. Then $\sigma$ can be extended to a null, geodesic and shear-free vector field $n$ on an open set in $M$.

Proof. The set of all integral curves of $\sigma$ is a 1-parameter family of null straight lines. This family determines the vector field $\sigma$ up to multiple. In terms of twistors, a real 1-dimensional curve $C$ in $\boldsymbol{P}^{3}(C)$ corresponds to this 1-parameter family of null lines. Take a holomorphic curve $\hat{C}$ in $\boldsymbol{P}^{3}(\boldsymbol{C})$ subject to $C \subset \hat{C}$ and extend $\hat{C}$ to a 2-dimensional holomorphic surface $S$ in $\boldsymbol{P}^{3}(\boldsymbol{C})$. Then we have $C \subset S \cap N$. The shear-free null congruence defined by $S \cap N$ is an extension of $C$. Therefore the vector field corresponding to $S \cap N$ are null, geodesic and shear-free. Among these we can choose one which extends $\sigma$.

Q.E.D.

\section{$\S 4$. Null strings and null electromagnetic fields}

In this section we prove the main theorems.

Let $\Sigma: x^{\mu}\left(\tau^{1}, \tau^{2}\right)$ be a null string and let $\sigma=\sigma^{\mu}\left(\partial / \partial x^{\mu}\right)$ be the vector field on $\Sigma$ subject to (1.3) and (1.4). For any point $x_{(0)}$ on $\Sigma, \sigma$ can be extended to a vector field $n=n^{\mu}\left(\partial / \partial x^{\mu}\right)$ which is defined on a neighbourhood of $x_{(0)}$ in $M$ and is null, geodesic, shear-free. Then there exists a neighbourhood $U_{0}$ of $x_{(0)}$ in $M$ and null electromagnetic field $F=F_{\mu \nu} d x^{\mu} \wedge d x^{\nu}$ defined on $U_{0}$ such that $F_{\mu \nu} n^{\nu}=0=* F_{\mu \nu} n^{\nu}$. Since $n=n^{\mu}\left(\partial / \partial x^{\mu}\right)$ coincides with $\sigma=\sigma^{\mu}\left(\partial / \partial x^{\mu}\right)$ on $\Sigma$, it follows that

$$
v_{\mu \nu}\left(\tau^{1}, \tau^{2}\right)-i * v_{\mu \nu}\left(\tau^{1}, \tau^{2}\right)=e^{w\left(\tau_{1}^{1}, \tau^{2}\right)}\left(F_{\mu \nu}\left(x\left(\tau^{1}, \tau^{2}\right)\right)-i * F_{\mu \nu}\left(x\left(\tau^{1}, \tau^{2}\right)\right)\right)
$$

with a complex function $w\left(\tau^{1}, \tau^{2}\right)$ on $U_{0}$. In the following we will show that we can take a null electromagnetic field such that $w\left(\tau^{1}, \tau^{2}\right)=0$. For this purpose we need some lemmas.

Throughout this section 2-dimensional surface $\Sigma: x^{\mu}\left(\tau^{1}, \tau^{2}\right)$ is a null string and its Plücker's coordinates are denoted by $v^{\mu \nu}$ :

$$
v^{\mu \nu}=x_{1}^{u} x_{2}^{\nu}-x_{1}^{\nu} x_{2}^{\mu}
$$


where $x_{i}^{\mu}=\partial x^{\mu} / \partial \tau^{i}, \mu=0,1,2,3$ and $i=1,2$.

By a direct calculation we have:

LEMMA 4.1. Plücker's coordinates $v^{\mu \nu}$ satisfy

$$
\frac{\partial v^{\mu \nu}}{\partial x_{i}^{\eta}}=\varepsilon^{i j} \delta^{\eta \mu} x_{j}^{\nu}+\varepsilon^{k i} \delta^{\eta \nu} x_{k}^{\mu} \quad \text { and } \quad v_{\mu \nu} \frac{\partial v^{\mu \nu}}{\partial x_{i}^{\eta}}=2 \varepsilon^{i k} v_{\eta \nu} x_{k c}^{\nu} .
$$

REMARK. We can also show

$$
* v_{\mu \nu} \frac{\partial v^{\mu \nu}}{\partial x_{i}^{\eta}}=2 \varepsilon^{i k_{*} * v_{\mu \nu} x_{k}^{\nu} .}
$$

Since the quantities $* v_{\mu \nu}$ are dual Plücker's coordinates of $\Sigma$, i.e., $* v_{\mu \nu} x_{k}^{\nu}=0$ for any $k$, we have

$$
* v_{\mu \nu} \frac{\partial v^{\mu \nu}}{\partial x_{i}^{\eta}}=0
$$

The null vector $\sigma=\sigma^{\mu}\left(\partial / \partial x^{\mu}\right)$ such that $v_{\mu \nu} \sigma^{\nu}=0=* v_{\mu \nu} \sigma^{\nu}$ has the expression $\sigma=\iota^{*} X$ where $\iota$ is the embedding of $\Sigma$ into $M$ and $X=X^{i}\left(\partial / \partial \tau^{i}\right)$ is the tangent vector of $\Sigma$ in $\left(\tau^{1}, \tau^{2}\right)$ space such that $g_{i j} X^{j}=0$.

LEMMA 4.2. The quantities $v_{\mu \nu}\left(\partial v^{\mu \nu} / \partial x_{j}^{\eta}\right)$ satisfy

$$
g_{i j}\left(v_{\mu \nu} \frac{\partial v^{\mu \nu}}{\partial x_{j}^{\eta}}\right)=0 .
$$

Proof. From Lemma 4.1 we have

$$
v_{\mu \nu} \frac{\partial v^{\mu \nu}}{\partial x_{j}^{\eta}}=2 \varepsilon^{j k} v_{\eta \nu} x_{k}^{\nu} .
$$

By virtue of

$$
v_{\eta \nu} x_{k}^{\nu}=\varepsilon^{h m} \frac{\partial x_{\eta}}{\partial \tau^{h}} \frac{\partial x_{\nu}}{\partial \tau^{m}} \frac{\partial x^{\nu}}{\partial \tau^{k}}=\varepsilon^{h m} g_{m k} \frac{\partial x_{\eta}}{\partial \tau^{h}},
$$

it folows that

$$
g_{i j}\left(v_{\mu \nu} \frac{\partial v^{\mu \nu}}{\partial x_{j}^{\eta}}\right)=2 \varepsilon^{j k} \varepsilon^{h m} g_{i j} g_{m k} \frac{\partial x_{\eta}}{\partial \tau^{h}}
$$

where

$$
\begin{aligned}
\varepsilon^{j k} g_{m k} \varepsilon^{h m} & =-\varepsilon^{j k} g_{k m} \varepsilon^{m h} \\
& =-\left[\begin{array}{rr}
0 & 1 \\
-1 & 0
\end{array}\right] \cdot\left[\begin{array}{ll}
g_{11} & g_{12} \\
g_{21} & g_{22}
\end{array}\right] \cdot\left[\begin{array}{rr}
0 & 1 \\
-1 & 0
\end{array}\right]=\left[\begin{array}{rr}
g_{22} & -g_{12} \\
-g_{21} & g_{11}
\end{array}\right]
\end{aligned}
$$


Therefore we find that

$$
g_{i j}\left(v_{\mu \nu} \frac{\partial v^{\mu \nu}}{\partial x_{j}^{\eta}}\right)=2 \operatorname{det}\left(g_{i j}\right) \delta_{i}^{k} \frac{\partial x_{\eta}}{\partial \tau^{k}}=0 .
$$

Q.E.D.

If $v_{\mu \nu}\left(\partial v^{\mu \nu} / \partial x_{i}^{\eta}\right)\left(\partial / \partial \tau^{i}\right)=0$ for all $\eta$, then we have $v_{\eta \nu} x_{k}^{\nu}=0$. With identities $* v_{\eta \nu} x_{k}^{\nu}=0$ we can verify that $\left(x_{1}^{\nu}\right)$ and $\left(x_{2}^{\nu}\right)$ are linearly dependent. This contradicts the assumption that $\Sigma$ is a 2-dimensional surface. Thus there exists $\eta$ such that $v_{\mu \nu}\left(\partial v^{\mu \nu} / \partial x_{i}^{\eta}\right)\left(\partial / \partial \tau^{i}\right)$ is not zero and parallel to $X^{i}\left(\partial / \partial \tau^{i}\right)$.

Furthermore, we need some algebraic identities.

Lemma 4.3. Let $c_{i j}, A_{\mu \nu}$ and $B^{\mu \nu}$ be defined by

$$
c_{i j}=a_{\mu}^{i} b_{j}^{\mu}, \quad A_{\mu \nu}=a_{\mu}^{1} a_{\nu}^{2}-a_{\nu}^{1} a_{\mu}^{2}, \quad B^{\mu \nu}=b_{1}^{\mu} b_{2}^{\nu}-b_{1}^{\nu} b_{2}^{\mu}
$$

where, $i, j=1,2, \mu=0,1,2,3$ and $a_{\mu}^{i}, b_{i}^{\mu}$ are independent variables. Then the identities

$$
\begin{aligned}
& B^{\{\mu \nu\}} \frac{\partial A_{\{\mu \nu\}}}{\partial a_{\alpha}^{i}}=b_{j}^{\alpha} \bar{c}^{j i}, \\
& A_{\{\mu \nu\}} \frac{\partial B^{\{\mu \nu\}}}{\partial b_{i}^{\alpha}}=a_{\alpha}^{j} \bar{c}^{i j}
\end{aligned}
$$

hold. Here $\{\mu \nu\}$ denotes $\mu<\nu$ component and $\bar{c}^{i j}$ is the algebraic complement of $c_{i j}$, i.e.,

$$
\left(\bar{c}^{i j}\right)=\left[\begin{array}{rr}
c_{22} & -c_{12} \\
-c_{21} & c_{11}
\end{array}\right] .
$$

Proof. It follows from Laplace's expansion formula that

$$
\operatorname{det}\left(c_{i j}\right)=A_{\{\mu \nu\}} B^{\{\mu \nu\}} .
$$

so we have

$$
\frac{\partial}{\partial a_{\alpha}^{i}} \operatorname{det}\left(c_{i j}\right)=B^{\{\mu \nu\}} \frac{\partial A_{\{\mu \nu\}}}{\partial a_{\alpha}^{i}} .
$$

On the other hand we find that

$$
\frac{\partial}{\partial a_{\alpha}^{i}} \operatorname{det}\left(c_{i j}\right)=\operatorname{Trace}\left(\bar{c}^{j k} \frac{\partial c_{k h}}{\partial a_{\alpha}^{i}}\right)=\bar{c}^{j k} \frac{\partial c_{k j}}{\partial a_{\alpha}^{i}}
$$

where 


$$
\frac{\partial c_{j h}}{\partial a_{\alpha}^{i}}=\frac{\partial}{\partial a_{\alpha}^{i}}\left(a_{\mu}^{j} b_{h}^{\mu}\right)=\delta_{i j} b_{h}^{\alpha}
$$

and that

$$
\frac{\partial}{\partial a_{\alpha}^{i}} \operatorname{det}\left(c_{i j}\right)=\bar{c}^{j k} \delta_{i k} b_{j}^{\alpha}=b_{j}^{\alpha} \bar{c}^{j i}
$$

Thus,

$$
B^{\{\mu \nu\}} \frac{\partial A_{\{\mu \nu\}}}{\partial a_{\alpha}^{i}}=b_{j}^{\alpha} \bar{c}^{j k} .
$$

Similarly we can prove (4.3).

Q.E.D.

Lemma 4.4. Let $c_{i j}\left(\tau^{1}, \tau^{2}\right)$ be a function defined by

$$
c_{i j}\left(\tau^{1}, \tau^{2}\right)=\frac{\partial S_{i}\left(x^{\mu}\left(\tau^{1}, \tau^{2}\right)\right)}{\partial x^{\mu}} \frac{\partial x^{\mu}}{\partial \tau^{j}}, \quad i, j=1,2,
$$

where $S_{i}$ is a function of $x^{\mu}$ and $x^{\mu}$ is a function of $\tau^{i}$. Then the algebraic complement $\bar{c}^{i j}$ of $c_{i j}$ satisfies

$$
\frac{\partial}{\partial \tau^{i}} \bar{c}^{i j}=0
$$

Proof. By a direct calculation we have

$$
\begin{aligned}
\frac{\partial}{\partial \tau^{i}} \bar{c}^{i 1} & =\frac{\partial}{\partial \tau^{1}} \bar{c}^{11}+\frac{\partial}{\partial \tau^{2}} \bar{c}^{21} \\
& =\frac{\partial}{\partial \tau^{1}} c_{22}-\frac{\partial}{\partial \tau^{2}} c_{21} \\
& =\frac{\partial}{\partial \tau^{1}}\left(\frac{\partial S_{2}(x)}{\partial x^{\mu}} \frac{\partial x^{\mu}}{\partial \tau^{2}}\right)-\frac{\partial}{\partial \tau^{2}}\left(\frac{\partial S_{2}(x)}{\partial x^{\mu}} \frac{\partial x^{\mu}}{\partial \tau^{1}}\right) \\
& =\frac{\partial^{2} S_{2}(x)}{\partial x^{\mu} \partial x^{\nu}} \frac{\partial x^{\nu}}{\partial \tau^{1}} \frac{\partial x^{\mu}}{\partial \tau^{2}}+\frac{\partial S_{2}(x)}{\partial x^{\mu}} \frac{\partial^{2} x^{\mu}}{\partial \tau^{1} \partial \tau^{2}}-\frac{\partial^{2} S_{2}(x)}{\partial x^{\mu} \partial x^{\nu}} \frac{\partial x^{\nu}}{\partial \tau^{2}} \frac{\partial x^{\mu}}{\partial \tau^{1}}-\frac{\partial S_{2}(x)}{\partial x^{\mu}} \frac{\partial^{2} x^{\mu}}{\partial \tau^{2} \partial \tau^{1}} \\
& =0 .
\end{aligned}
$$

Similarly we have

$$
\frac{\partial}{\partial \tau^{i}} \bar{c}^{i 2}=0
$$

Q.E.D. 
The next proposition is well known (see, for example, Godbillon [3]).

Proposition 4.5. Let $F$ be a differential 2-form on an open set $U$ in $M$ such that $d F=0$ and $F \wedge F=0$. Then for any point $x_{(0)}$ in $U$, there exists a neighbourhood $U_{0}$ of $x_{(0)}$ and functions $S_{i}(x), i=1,2$ defined on $U_{0}$ such that

$$
F=d S_{1} \wedge d S_{2} \quad \text { on } U_{0} .
$$

REMARK. Since $F \wedge F=-* F \wedge * F=(1 / 4) \eta(F, * F) d x^{0} \wedge d x^{1} \wedge d x^{2} \wedge d x^{3}$, null electromagnetic fields satisfy the conditions of Proposition 4.5. Thus differential 2-forms $F$ and $* F$ have the forms

$$
F=d S_{1} \wedge d S_{2} \text { and } * F=d T_{1} \wedge d T_{2}
$$

with certain functions $S_{1}, S_{2}$ and $T_{1}, T_{2}$.

LEMma 4.6. Let $\Sigma: x^{\mu}\left(\tau^{1}, \tau^{2}\right)$ be an analytic null string and let $x_{(0)}$ be any point on $\Sigma$. Then there exist a neighbourhood $U_{0}$ of $x_{(0)}$ in $M$ and a null electromagnetic field $F=F_{\mu \nu} d x^{\mu} \wedge d x^{\nu}$ defined on $U_{0}$ such that

$$
v^{\mu \nu}\left(\tau^{1}, \tau^{2}\right)-i * v^{\mu \nu}\left(\tau^{1}, \tau^{2}\right)=e^{w\left(\tau^{1}, \tau^{2}\right)}\left(F^{\mu \nu}\left(x\left(\tau^{1}, \tau^{2}\right)\right)-i * F^{\mu \nu}\left(x\left(\tau^{1}, \tau^{2}\right)\right)\right)
$$

with an appropriate complex function $w\left(\tau^{1}, \tau^{2}\right)$ is valid on $U_{0}$. Furthermore, the equation

$$
X^{i} \frac{\partial}{\partial \tau^{i}} w\left(\tau^{1}, \tau^{2}\right)=0
$$

holds, where $X=X^{i}\left(\partial / \partial \tau^{i}\right)$ is a tangent vector of $\Sigma$ such that $\sigma=\iota^{*} X$

Proof. The first statement was already shown. The second statement is proved as follows.

The Euler-Largrange equations for string motion are

$$
\frac{\partial}{\partial \tau^{j}}\left(v_{\{\mu \nu\}} \frac{\partial v^{\{\mu \nu\}}}{\partial x_{j}^{\gamma}}\right)=0,
$$

where $\{\mu \nu\}$ denotes $\mu<\nu$ component. Using the identities (4.1), we have

$$
\frac{\partial}{\partial \tau^{j}}\left\{\left(v_{\{\mu \nu\}}-i * v_{\{\mu \nu\}}\right) \frac{\partial v^{\{\mu \nu\}}}{\partial x_{j}^{\eta}}\right\}=0 .
$$

Substituting (4.4) into (4.6), 


$$
\begin{aligned}
0 & =\frac{\partial}{\partial \tau^{j}}\left\{e^{w\left(\tau^{1}, \tau^{2}\right)}\left(F_{\{\mu \nu\}}-i * F_{\{\mu \nu]}\right) \frac{\partial v^{\{\mu \nu\}}}{\partial x_{j}^{\eta}}\right\} \\
& =\left(F_{\{\mu \nu\}}-i * F_{\{\mu \nu\}}\right) \frac{\partial v^{\{\mu \nu\}}}{\partial x_{j}^{\eta}} e^{w\left(\tau^{1}, \tau^{2}\right)} \frac{\partial}{\partial \tau^{j}} w\left(\tau^{1}, \tau^{2}\right)+e^{w\left(\tau^{1}, \tau^{2}\right)} \frac{\partial}{\partial \tau^{j}}\left\{\left(F_{\{\mu \nu\}}-i * F_{\{\mu \nu\}}\right) \frac{\partial v^{\{\mu \nu\}}}{\partial x_{j}^{\eta}}\right\} \\
& =\left(v_{\{\mu \nu\}}-i * v_{\{\mu \nu\}}\right) \frac{\partial v^{\mu \nu}}{\partial x_{j}^{\eta}} \frac{\partial}{\partial \tau^{j}} w\left(\tau^{1}, \tau^{2}\right)+e^{w\left(\tau^{1}, \tau^{2}\right)} \frac{\partial}{\partial \tau^{j}}\left\{\left(F_{\{\mu \nu\}}-i * F_{\{\mu \nu\}}\right) \frac{\partial v^{\{\mu \nu\}}}{\partial x_{j}^{\eta}}\right\} \\
(4.7) & =v_{\{\mu \nu\}} \frac{\partial v^{\{\mu \nu\}}}{\partial x_{j}^{\eta}} \frac{\partial}{\partial \tau^{j}} w\left(\tau^{1}, \tau^{2}\right)+e^{w\left(\tau^{1}, \tau^{2}\right)} \frac{\partial}{\partial \tau^{j}}\left\{\left(F_{\{\mu \nu\}}-i * F_{\{\mu \nu\}}\right) \frac{\partial v^{\{\mu \nu\}}}{\partial x_{j}^{\eta}}\right\} .
\end{aligned}
$$

Next we show

$$
\frac{\partial}{\partial \tau^{j}}\left(F_{\{\mu \nu\}} \frac{\partial v^{\{\mu \nu\}}}{\partial x_{j}^{\eta}}\right)=0
$$

and

$$
\frac{\partial}{\partial \tau^{j}}\left(* F_{\{\mu \nu\}} \frac{\partial v^{\{\mu \nu\}}}{\partial x_{j}^{\eta}}\right)=0 .
$$

To prove (4.8) we represent $F_{\{\mu \nu\}}$ using Proposition 4.5 as follows:

$$
F_{\{\mu \nu\}}=s_{\{\mu \nu\}}
$$

where

$$
s_{\{\mu \nu\}}=S_{1 \mu}(x) S_{2 \nu}(x)-S_{1 \nu}(x) S_{2 \mu}(x)
$$

and $S_{i \mu}(x)=\left(\partial S_{i} / \partial x^{\mu}\right)$. Using this representation of $F_{\{\mu \nu\}}$ we have

$$
\frac{\partial}{\partial \tau^{j}}\left(F_{\{\mu \nu\}} \frac{\partial v^{\{\mu \nu\}}}{\partial x_{j}^{\eta}}\right)=\frac{\partial}{\partial \tau^{j}}\left(s_{\{\mu \nu\}} \frac{\partial v^{\{\mu \nu\}}}{\partial x_{j}^{\eta}}\right) .
$$

Lemma 4.3 implies that

$$
\frac{\partial}{\partial \tau^{j}}\left(s_{\{\mu \nu\}} \frac{\partial v^{\{\mu \nu\}}}{\partial x_{j}^{\eta}}\right)=\frac{\partial}{\partial \tau^{j}}\left(S_{k \eta} \bar{c}^{j k}\right)
$$

and Lemma 4.4 implies that

$$
\begin{aligned}
\frac{\partial}{\partial \tau^{j}}\left(S_{k \eta} \bar{c}^{j k}\right) & =\bar{c}^{j k} \frac{\partial}{\partial \tau^{j}} S_{k \eta}+S_{k \eta} \frac{\partial}{\partial \tau^{j}} \bar{c}^{j k} \\
& =\bar{c}^{j k} \frac{\partial}{\partial \tau^{j}} S_{k \eta} .
\end{aligned}
$$

Further we have from Lemma 4.3 


$$
\bar{c}^{j k} \frac{\partial}{\partial \tau^{j}} S_{k \eta}=\frac{\partial^{2} S_{k}}{\partial x^{\eta} \partial x^{\rho}} \frac{\partial x^{\rho}}{\partial \tau^{j}} \bar{c}^{j k}=\frac{\partial^{2} S_{k}}{\partial x^{\eta} \partial x^{\rho}} v^{\{\mu \nu\}} \frac{\partial F_{\{\mu \nu\}}}{\partial S_{k \rho}} .
$$

Since the identities

$$
* F^{\{\mu \nu\}} \frac{\partial F_{\{\mu \nu\}}}{\partial S_{k \rho}}=0
$$

hold, we find that

$$
\begin{aligned}
\bar{c}^{j k} \frac{\partial}{\partial \tau^{j}} S_{k \eta} & =\frac{\partial^{2} S_{k}}{\partial x^{\eta} \partial x^{\rho}} v^{\{\mu \nu\}} \frac{\partial F_{\{\mu \nu\}}}{\partial S_{k \rho}}-(i / 2)\left(e^{\bar{w}}-e^{w}\right) \frac{\partial^{2} S_{k}}{\partial x^{\eta} \partial x^{\rho}} * F^{\{\mu \nu\}} \frac{\partial F_{\{\mu \nu\}}}{\partial S_{k \rho}} \\
& =v^{\{\mu \nu\}} \frac{\partial F_{\{\mu \nu\}}}{\partial x^{\eta}}-(i / 2)\left(e^{\bar{w}}-e^{w}\right) * F^{\{\mu \nu\}} \frac{\partial F_{\{\mu \nu\}}}{\partial x^{\eta}} \\
& =(1 / 2)\left(e^{w}+e^{\bar{w}}\right) F^{\{\mu \nu\}} \frac{\partial F_{\{\mu \nu\}}}{\partial x^{\mu}}=0 .
\end{aligned}
$$

The last equality follows from $F^{\{\mu \nu\}} F_{\{\mu \nu\}}=0$. Thus we prove (4.8). Similarly, using $* F=d T_{1} \wedge d T_{2}$, we can prove (4.9). Substituting (4.8) and (4.9) into (4.7), we have

$$
v_{\{\mu \nu\}} \frac{\partial v^{\{\mu \nu\}}}{\partial x_{j}^{\eta}} \frac{\partial}{\partial \tau^{j}} w\left(\tau^{1}, \tau^{2}\right)=0 .
$$

Therefore by Lemma 4.2, we obtain

$$
X^{j} \frac{\partial}{\partial \tau^{j}} w\left(\tau^{1}, \tau^{2}\right)=0
$$

Q.E.D.

From the proof of Lemma 4.6, we have:

Theorem 4.7. Let $F=F_{\mu \nu} d x^{\mu} \wedge d x^{\nu}$ be a closed null 2-form on $M$ and let $\Sigma: x^{\mu}\left(\tau^{1}, \tau^{2}\right)$ be a 2-dimensional surface such that $v^{\mu \nu}\left(\tau^{1}, \tau^{2}\right)=F^{\mu \nu}\left(x\left(\tau^{1}, \tau^{2}\right)\right)$ on $\Sigma$. Then $\Sigma$ is a null string.

REMARK. Let $F$ be a null electromagnetic field. Then there exist functions $T_{1}(x), T_{2}(x)$ such that $* F=d T_{1} \wedge d T_{2}$. Let $\Sigma: x^{\mu}\left(\tau^{1}, \tau^{2}\right)$ be a surface defined by $T_{i}=c_{i}(i=1,2), c_{i}$ being constants. Then we have

$$
* F_{\mu \nu}(x) \frac{\partial x^{\nu}}{\partial \tau^{1}}=0 \quad \text { and } \quad * F_{\mu \nu}(x) \frac{\partial x^{\nu}}{\partial \tau^{2}}=0 .
$$

Therefore $v^{\mu \nu}\left(\tau^{1}, \tau^{2}\right)=g\left(\tau^{1}, \tau^{2}\right) F^{\mu \nu}\left(x\left(\tau^{1}, \tau^{2}\right)\right)$ with a function $g\left(\tau^{1}, \tau^{2}\right)$. After the coordinate transformation subject to $\operatorname{det}\left(\partial \hat{\tau}^{i} / \partial \tau^{j}\right)=g\left(\tau^{1}, \tau^{2}\right)$, Plücker's coordinates $v^{\mu \nu}\left(\hat{\tau}^{1}, \hat{\tau}^{2}\right)$ satisfy 


$$
v^{\mu \nu}\left(\hat{\tau}^{1}, \hat{\tau}^{2}\right)=\operatorname{det}\left(\frac{\partial \tau^{i}}{\partial \hat{\tau}^{j}}\right) v^{\mu \nu}\left(\tau^{1}, \tau^{2}\right)=F^{\mu \nu}\left(x\left(\hat{\tau}^{1}, \hat{\tau}^{2}\right)\right)
$$

So $\Sigma: x^{\mu}\left(\tau^{1}, \tau^{2}\right)$ is a null string.

Finally we prove that the complex function $w\left(\tau^{1}, \tau^{2}\right)$ in Lemma 4.6 can be extended to a complex function $w\left(x^{\mu}\right)$ on an open set in $M$ subject to (2.12) and (2.13). Then under a transformation (2.3) defined by $w\left(x^{\mu}\right)$, the transformed 2 -form is a null electromagnetic field.

Let $\left(x^{\lambda}, x^{3}\right), \lambda=0,1,2$ be a local coordinate system of $M$ such that $n^{\mu}=$ $\delta_{3}^{\mu}$. By taking coordinates $\left(\tau^{1}, \tau^{2}\right)$ on $\Sigma$ such that

$$
\frac{\partial}{\partial \tau^{1}}=\sigma
$$

we define a vector field $k^{\mu}$ on $\Sigma$ by $k^{\mu}=\partial x^{\mu} / \partial \tau^{2}$. Using the quasi-orthonormal basis in Proposition 2.1, the vector $k^{\mu}$ is represented as follows:

$$
k^{\mu}=f n^{\mu}+g m^{\mu}+\bar{g} \bar{m}^{\mu}
$$

where $f$ is a real function and $g$ is a complex function. We see from (4.12) and $\sigma^{\mu}=\partial x^{\mu} / \partial \tau^{1}=\delta_{3}^{\mu}$ that $x^{0}\left(\tau^{1}, \tau^{2}\right), x^{1}\left(\tau^{1}, \tau^{2}\right)$ and $x^{2}\left(\tau^{1}, \tau^{2}\right)$ are independent of $\tau^{1}$. We denote these by $x^{0}\left(\tau^{2}\right), x^{1}\left(\tau^{2}\right)$ and $x^{2}\left(\tau^{2}\right)$, respectively. Also we have $x^{3}\left(\tau^{1}, \tau^{2}\right)$ $=\tau^{1}+h\left(\tau^{2}\right)$ with a function $h\left(\tau^{2}\right)$. Thus, after a coordinate transformation $\tau^{1}=\tau^{1}+h\left(\tau^{2}\right), \tau^{2}=\tau^{2}$, we can assume that $\Sigma$ is represented in $M$ by

$$
\Sigma:\left(x^{0}\left(\tau^{2}\right), x^{1}\left(\tau^{2}\right), x^{2}\left(\tau^{2}\right), \tau^{1}\right)
$$

Let $w\left(\tau^{1}, \tau^{2}\right)$ be the function in Lemma 4.6. The function $w\left(\tau^{1}, \tau^{2}\right)$ satisfies

$$
\frac{\partial}{\partial \tau^{1}} w\left(\tau^{1}, \tau^{2}\right)=0
$$

Therefore $w\left(\tau^{1}, \tau^{2}\right)$ is independent of $\tau^{1}$ and we denote this by $w_{0}\left(\tau^{2}\right)$. We define a curve in $\left(x^{\lambda}\right)$ space of constant $x^{3}$ by

$$
c\left(\tau^{2}\right)=\left(x^{0}\left(\tau^{2}\right), x^{1}\left(\tau^{2}\right), x^{2}\left(\tau^{2}\right)\right) .
$$

The tangent vector of $c\left(\tau^{2}\right)$ is $k^{2}=g m^{2}+\bar{g} \bar{m}^{2}$ where the complex function $g$ is not zero and $\lambda=0,1,2$.

LEMMA 4.8. The vectors $k^{\lambda}$ and $m^{\lambda}$ are linearly independent.

Proof. If $k^{\lambda}$ and $m^{\lambda}$ are linearly dependent, then there exists a function $h$ such that $k^{\lambda}=h m^{\lambda}, \lambda=0,1,2$. Since $n^{\mu} m_{\mu}=0$ and $n^{\mu}=\delta_{3}^{\mu}$, we see $m_{3}=0$. Therefore we have 


$$
m^{\lambda} m_{\lambda}=m^{\lambda} m_{\lambda}+m^{3} m_{3}=m^{\mu} m_{\mu}=0
$$

and

$$
k^{\lambda} m_{\lambda}=k^{\lambda} m_{\lambda}+k^{3} m_{3}=k^{\mu} m_{\mu}=-\bar{g} .
$$

Thus we have

$$
-\bar{g}=k^{\lambda} m_{\lambda}=h m^{2} m_{\lambda}=0 .
$$

This contradicts the fact that $g$ is not zero.

Q.E.D.

If we admit complex values for $\tau^{2}$, we have a holomorphic curve $\hat{c}$ in $C^{3}$. We can take a holomorphic 2-dimensional surface $S$ in $C^{3}$ such that $\hat{c} \subset S$ and is transversal to $m^{2}$. Let $\hat{w}_{0}$ be a holomorphic function on $S$ which coincides with $w_{0}$ on $c$. Let $\hat{w}$ be a solution of equation (2.13) on $\left(x^{\lambda}\right)$ space of constant $x^{3}$, which in this case takes the form

$$
\hat{w},{ }_{\lambda} m^{\lambda}=0
$$

with initial value $\hat{w}_{0}$ on $S$. The solution $\hat{w}$ exists and coincides with $w_{0}\left(\tau^{2}\right)$ on $c$, i.e., $\hat{w}=w$ on $\Sigma$. Under transformation (2.3) defined by $w\left(x^{\mu}\right)$, the transformed 2 -form is a null electromagnetic field. Thus we have:

THEOREM 4.9. Let a 2-dimensional surface $\Sigma: x^{\mu}\left(\tau^{1}, \tau^{2}\right)$ in Minkowski space be an analytic null string and let $x_{(0)}$ be a point on $\Sigma$. Then a neighbourhood $U_{0}$ of $x_{(0)}$ in Minkowski space and a null electromagnetic field $F=$ $F_{\mu \nu} d x^{\mu} \wedge d x^{\nu}$ defined on $U_{0}$ such that

$$
v^{\mu \nu}\left(\tau^{1}, \tau^{2}\right)=F^{\mu \nu}\left(x\left(\tau^{1}, \tau^{2}\right)\right), \quad x\left(\tau^{1}, \tau^{2}\right)=\left(x^{\mu}\left(\tau^{1}, \tau^{2}\right)\right) \in U_{0}
$$

exist, where Plücker's coordinates $v^{\mu \nu}$ of $\Sigma$ are defined by

$$
v^{\mu \nu}=\frac{\partial x^{\mu}}{\partial \tau^{1}} \frac{\partial x^{\nu}}{\partial \tau^{2}}-\frac{\partial x^{\nu}}{\partial \tau^{1}} \frac{\partial x^{\mu}}{\partial \tau^{2}} .
$$

\section{References}

[1] C. Carathéodory, Utber der Varitionsrechnung bei mehrfachen Integralen, Acta Sci. Math. (Szeged), 4 (1929), 193-216, Reprinted in: C. Carathéodory: Gesamm. Math. Schriften, Vol. 1, 401-426. München: Beck 1954.

[2] D. Cox and E. J. Flaherty, A conventional proof of Kerr's theorem, Commu. Math. Phys., 47 (1976), 75-79.

[ 3 ] C. Godbillon, Geometrie differentielle et mechanique, Chap. VI, Hermann 1969.

[4] A. I. Janis and E. T. Newman, Structure of Gravitational sources, J. Math. Phys., 6 (1965), 902-914.

[ 5 ] K. Nagatomo, On a construction of null electromagnetic fields, Osaka J. Math., 20 (1983), 285-301. 
[6] E. Newman and R. Penrose, An approach to gravitational radiation by a method of spin coefficients, J. Math. Phys., 3 (1962), 566-578.

[7] H. A. Kastrup, On the relation of Regge-trajectories to degenerate classical solutions of equations of motion, Phys. Lett., 78(B) (1978), 433-437.

[ 8 ] H. A. Kastrup, Relativistic strings and electromagnetic flux tubes, Phys. Lett., 82 (B) (1979), 237-238.

[ 9 ] H. A. Kastrup and M. Rinke, Hamilton-Jacobi theory for strings, Phys. Lett., 105 (B) (1981), 191-196.

[10] R. Penrose, Twistor algebra, J. Math. Phys., 3 (1967), 345-366.

[11] M. Rinke, The relation between relativistic strings and Maxwell fields of rank 2, Comm. Math. Phys., 73 (1980), 265-271.

[12] I. Robinson, Null electromagnetic fields, J. Math. Phys., 2 (1961), 290-291.

[13] H. Rund, The Hamilton-Jacobi theory in the calculus of variations, Chap. 4, D. Van Nostrand, 1966.

[14] R. Sachs, Gravitational waves in general relativity VI. The outgoing radiation condition, Proc. Roy. Soc. London Ser. A, 264 (1961), 309-338.

[15] A. Schild, Classical null strings, Phys. Rev. D, 16 (1977), 1722-1726.

[16] W. Velte, Zur Variationsrechung mehrfacher Integrale, Math. Z., 60 (1954), 367-383.

[17] R. O. Wells, Jr., Complex manifolds and mathematical physics, Bull. Amer. Math. Soc. (New Series), 1 (1979), 296-336.

\author{
KiYoKaZU Nagatomo \\ Department OF MATHEMATICS \\ FACULTY OF ScIENCE \\ OSAKA UNIVERSITY \\ TOYONAKA, OSAKA 560 \\ JAPAN
}

